\title{
COMMUNICATIONS
}

\section{EXPERIMENTAL AND CLINICAL OBSERVATIONS ON THE ECHOGRAMS IN VITREOUS HAEMORRHAGES*†}

\author{
BY
}

ARVO OKSALA

From the Ophthalmic Department of the University Hospital, Turku, Finland

IN previous communications (Oksala and Lehtinen, 1959; Oksala 1960a, b), I noted that vitreous haemorrhages reflected echoes on the screen of an ultrasound equipment, and that these echoes were clearer if a fresh haemorrhage was involved. I also found that old haemorrhages, on account of the destruction of the vitreous, do not always become visible in ultrasonic examinations (Oksala and Lehtinen, 1959). Baum and Greenwood (1958), who have also noted clear echoes in vitreous haemorrhages, stated that it was possible to differentiate between them and the echoes obtained from a foreign body.

During these last 2 years I have been better able to visualize echoes from vitreous haemorrhages by using a more sensitive crystal, which has also made possible so-called "selective echography". In the present paper I propose to describe my observations on vitreous haemorrhages with the new instruments, which show an improvement in the possibilities of diagnosis by ultrasound.

\section{Research Equipment}

I have continued to use the ultrasonic equipment manufactured by Dr. J. u. H. Krautkrämer, Mode. Usip. 9. At my request this same factory manufactured a specially sensitive barium titanate crystal with a slight damping, a frequency of $6 \mathrm{MHz}$ and a diameter of $10 \mathrm{~mm}$. Resolution in depth was about $0.6 \mathrm{~mm}$. in the eye. With this crystal echoes could be obtained even from slight vitreous haemorrhages.

Another ultrasonic instrument was constructed $\ddagger$ using a barium titanate crystal with a frequency of $10 \mathrm{MHz}$ which also proved to have a very "broad spectrum".

The sensitive "broad spectrum" crystal enabled me to use the Krautkrämer ultrasonic equipment for the selective echography which I have used for about 2 years in differential diagnosis. By reducing the power of the issuing impulse (IP) as well as the amplification of the returning impulse, it is possible to differentiate between various intra-ocular pathological changes because they produce acoustic reactions of different density. These results have been described elsewhere (Oksala, 1962). The IP values indicated do not represent fixed measuring units, as the IP can be regulated gradually from 1 to 5 and the amplification without gradation from 1 to 10 .

* Received for publication August 7, 1962.

+ This study was supported by research grant of the Sigrid Jusélius Stiftelse.

$\ddagger$ By Kretztechnik, Zipf, Austria.

5 
Since a fresh haemorrhage into the vitreous causes the formation of vesicles, the echograms reflected by uncoagulated heparin blood were investigated and also the effect of ultrasound absorption in the cornea and sclera on the amplitude of the echoes.

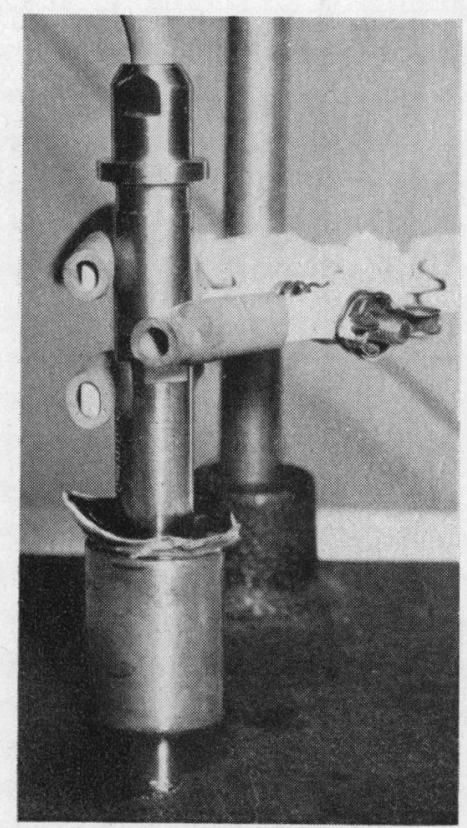

FIG. 1.-Method of experimental investigation. The metal container is filled with heparin blood. The crystal fixed to a stand is in contact with the blood through the mediation of the scleral tissue.

Heparin blood was poured into a metal container and the crystal was fixed to a stand and pressed against the surface of the liquid. The echograms so obtained were photographed. Calotte-like pieces of cornea and sclera were cut from a bovine eye, and these pieces were placed in turn on the container so that they touched the surface of the fluid. When the crystal was then pressed against the cornea and the sclera in turn (as shown in Fig. 1), echograms were again seen on the screen and the effects of corneal and scleral absorption could be studied by selective echography.

\section{Clinical Material}

Ten cases each of fresh and old vitreous haemorrhage were studied. The results obtained in each group were similar and three typical instances are described below.

\section{Results}

\section{Experimental}

(a) Echogram of Uncoagulated Blood.-Fig. 2 illustrates echograms obtained from uncoagulated blood, on the left a high initial impulse, and on the right high echoes reflected by the bottom of the container. Between them there is a group of lower echoes emitted by the uncoagulated blood. In Fig. 2A the IP was 1 and the amplification was 10, when the blood reflected clear echoes with a fairly similar amplitude. When the amplification was reduced to 6 the echoes were only just visible (Fig. 2B).

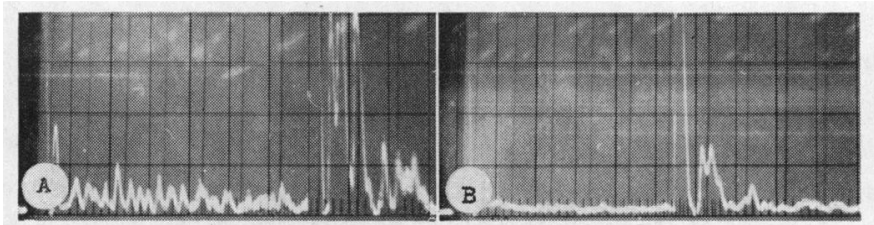

FIG. 2.-Experimental echograms obtained by pressing the crystal direct against the blood. On the left there is the high initial impulse and on the right the high echoes from the bottom of the container. In between are the echoes reflected by the blood.

(A) IP 1 and amplification 10.

(B) IP 1 and amplification 6. Here the reflections are seen as very low echoes. 
(b) Echogram altered by Interposition of Tissue from Cornea or Sclera.When tissue from the cornea and the sclera in turn was interposed between the crystal and the blood, the echograms illustrated in Fig. 3 were obtained. In Fig. 3A, the cornea was interposed between the crystal and the blood, with the IP 1 and the amplification 10; the echogram was similar to those in Fig. 2A. Fig. 3B shows the echogram when the cornea was interposed between the crystal and the blood, with IP 1 and the amplification 6 . In Fig. 3C the sclera was interposed between the crystal and the blood, with IP 1 and the amplification 6. The echograms in Figs 3B and C show that the cornea and the sclera absorb the ultrasound to such an extent that, if the IP is 1 and the amplification 6, the echoes of uncoagulated blood almost disappear. The differences compared to the results in Fig. 2B are slight but none the less distinct. The same result was obtained when the experiment was repeated several times.

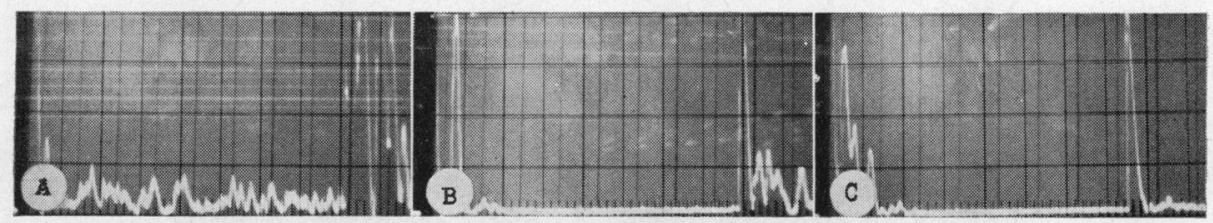

Fig. 3.-Experimental echograms obtained when the cornea and the sclera were interposed in turn between the crystal and the blood.

(A) The cornea was between the crystal and the blood, with IP 1 and amplification 10.

(B) The amplification was changed to 6.

(C) The sclera was between the crystal and the blood, with IP 1 and amplification 6.

In (B) and (C) the echoes reflected by the blood are hardly visible.

\section{Clinical}

Case 1.-This patient had a sudden onset of extensive vitreous haemorrhage in one eye for the first time. The visual acuity was reduced to hand movements and the red reflex was not obtained. Fig. 4 shows two echograms obtained in the ultrasonic examination.

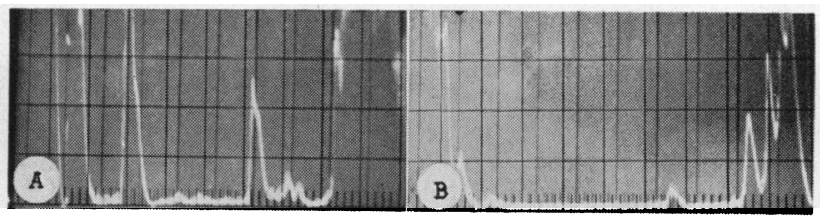

FIG. 4. - Case 1, a first fresh haemorrhage.

(A) The sound wave was directed axially, and echoes from the lens were also seen. The vitreous space emitted one higher and several lower echoes, with IP 3 and amplification 10.

(B) There was only one echo from the vitreous haemorrhage, with IP 1 and amplification 10.

In Fig. 4A the IP was 3 and the amplification 10. The initial impulse on the left is followed by high echoes reflected from the anterior and posterior surface of the lens. The vitreous space emitted one higher and several lower echoes. The echoes on the right came from the posterior wall of the eye and the retrobulbar tissue. I am of the opinion that one echo reflected 
from the vitreous which was clearly higher than the rest came from the border surface between the vitreous and a large blood vesicle.

In Fig. 4B the IP was 1 and the amplification 10, and only this high echo was emitted by the vitreous. It disappeared again when the amplification was reduced to 6 . In Fig. 4B the crystal was not guided quite axially.

Case 2.-This patient had had repeated vitreous haemorrhages in one eye for a number of years, the last 2 weeks before the ultrasound examination. The visual acuity was hand movements and the red reflex was absent. Fig. 5 shows two echograms. In Fig. 5A the IP was 5 and the amplification 10, and abundant low echoes were reflected from the whole vitreous space. In Fig. 5B the IP was 5 and the amplification 5, and low echoes from the vitreous could still be seen, but no echoes were seen if the IP was 1 and the amplification was 6.

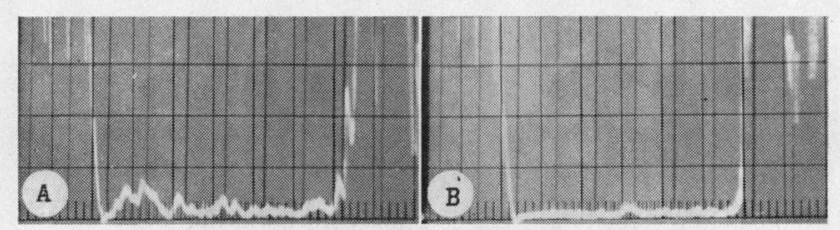

Fig. 5.-Case 2, a recent vitreous haemorrhage after several previous ones.

(A) Numerous echoes reflected by the vitreous space, with IP 5 and amplification 10.

(B) The vitreous space reflects very low echoes, with IP 5 and amplification 5.

This patient was also examined by the Kretztechnik ultrasound equipment with a frequency of $10 \mathrm{MHz}$. Fig. 6A shows normal echoes obtained from the healthy eye. Here a pure zero line lies between the anterior and posterior surface of the eye, i.e. no echoes were reflected from the vitreous. Fig. 6B illustrates the echogram obtained from the affected eye, in which numerous echoes were reflected from the vitreous space.

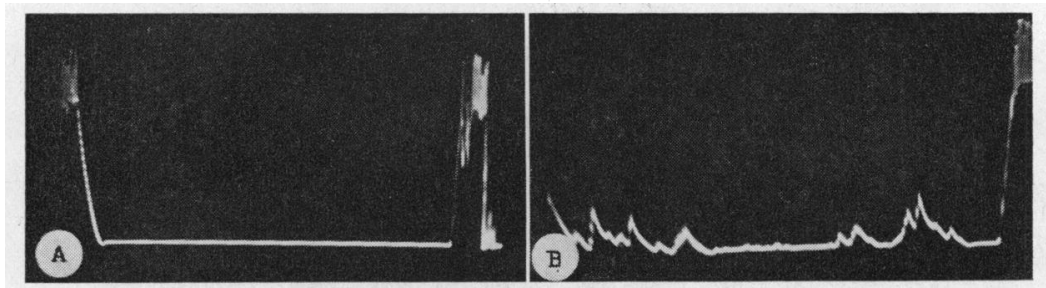

Fig. 6.-Case 2, examined with the Kretztechnik ultrasonic equipment at a frequency of $10 \mathrm{MHz}$.

(A) The healthy vitreous is acoustically fully homogeneous.

(B) With the same equipment, the vitreous space of the affected eye reflected numerous echoes.

Case 3.-This patient had had repeated vitreous haemorrhages in one eye during 2 years, and the ultrasonic examination was carried out 2 weeks after 
the most recent haemorrhage. The visual acuity was then hand movements and the red reflex was not obtained.

Ultrasonic examination was performed with the Kretztechnik equipment at a frequency of $10 \mathrm{MHz}$. Fig. 7A shows the echogram obtained from the healthy eye in the axial direction. The echoes on the left are from the initial impulse and the anterior surface of the lens; the following higher echoes come from the posterior surface of the lens, the vitreous is represented by a zero line and the echoes on the right come from the posterior surface of the eye and the retrobulbar tissue.

Fig. 7B illustrates the echogram obtained from the affected eye; numerous low echoes came from the vitreous space.

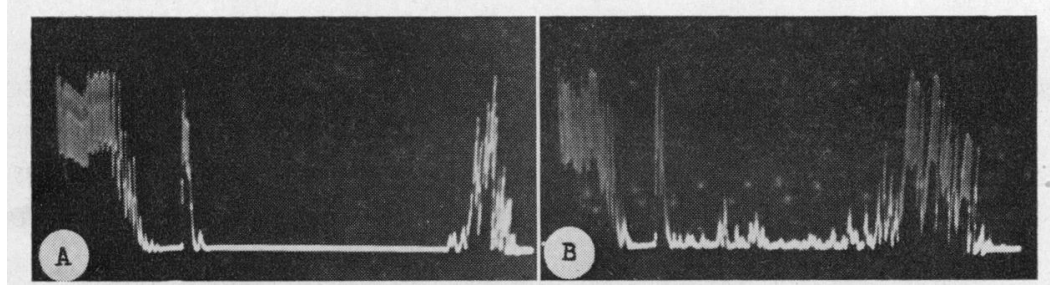

Fig. 7.-Case 3, a recent vitreous haemorrhage after several previous ones, examined with the Kretztechnik equipment at a frequency of $10 \mathrm{MHZ}$.

(A) The echogram of the sound eye examined axially shows on the left the initial impulse and echoes from the anterior surface of the lens. The subsequent high echo group is reflected by the posterior surface of the lens. No echoes are emitted by the vitreous space.

(B) The affected eye, also examined axially shows several low echoes reflected by the vitreous space.

\section{Discussion}

The experimental study showed that uncoagulated blood is acoustically non-homogeneous. If there is a large blood vesicle in the eye, several low echoes are obtained from within the vesicle in addition to echoes from both border surfaces. Corneal and scleral absorption was so high in the experimental study, that the results of selective echography in the experimental and clinical investigations were highly consistent with each other.

With our present equipment it is possible to diagnose very slight vitreous haemorrhages solely on the basis of echograms. This is of great clinical significance if we wish to examine the vitreous space when opacities of the cornea or lens prevent the use of the usual optical aids.

If the vitreous is mainly undamaged, the extensive and clear border surfaces between the vitreous and the haemorrhage give back high echoes. Although a fresh haemorrhage usually reflects one or two higher echoes, the time of occurrence of a haemorrhage, e.g., in eyes affected with cataract, cannot be reliably assessed on the basis of echograms only.

Intra-ocular inflammations produce vitreous opacities which usually also reflect echoes, and it is not possible to distinguish vitreous haemorrhages from vitreous opacities produced by inflammation by echograms only. 
On the other hand, selective echography differentiates with considerable accuracy between an intra-ocular foreign body, a tumour, a retinal detachment, and a vitreous haemorrhage. All echoes from a vitreous haemorrhage disappear when the IP is 1 and the amplification 6, but those from the other pathological changes disappear only when the IP is 1 and the amplification is less than 3. This resolving threshold is so high that false positive or negative results in the clinical examination are rare.

Both the ultrasonic instruments demonstrated the vitreous haemorrhages equally well on both 6 and $10 \mathrm{MHz}$.

\section{Summary}

The echograms reflected by vitreous haemorrhages have been studied experimentally and clinically. Even fresh uncoagulated blood reflects low echoes. The amplitude is affected to some extent by corneal and scleral absorption.

In clinical investigations fresh vitreous haemorrhages reflected one or two higher echoes, whereas old haemorrhages gave only low echoes because of the destruction of the vitreous.

Selective echography permits the differential diagnosis of vitreous haemorrhages, intra-ocular foreign bodies, and tumours, as well as retinal detachments.

On the other hand, vitreous haemorrhages cannot be reliably distinguished from vitreous opacities due to inflammation.

\section{REFERENCES}

Baum, G., and Greenwood, I. (1958). A.M.A. Arch. Ophthal., 60, 263.

OKSALA, A. (1960). Amer. J. Ophthal., 49, 1301.

(1960). Klin. Mbl. Augenheilk., 137, 72.

(1962). Acta ophthal. (Kbh.), 40, in the press.

and Lehtinen, A. (1959). Ibid., 37, 17. 